\title{
Population Genetics of Heterosis: Effects of Hardy-Weinberg Disequilibrium
}

\author{
By R. Wu ${ }^{1,2, *}$, CH.-X. MA ${ }^{2}$, J. Wu ${ }^{1}$, W. FANG ${ }^{1}$ and G. CASELla ${ }^{2}$ \\ Department of Statistics, University of Florida, Gainesville, FL 32611
}

(Received 22 ${ }^{\text {rd }}$ April 2003)

\begin{abstract}
Summary
The mechanistic explanation of heterosis has been traditionally based on quantitative differences of gene effects. However, for outcrossing populations, heterosis is also a property of populations. In this paper, the effects of the deviation of a population from Hardy-Weinberg equilibrium (HWE) on the magnitude of heterosis have been examined numerically. The mating of two populations in HWE may generate directions and magnitudes of heterosis different from the mating of two populations in Hardy-Weinberg disequilibrium (HWD). Such differences of heterosis between these two types of population mating may be due to the release of vigor restored in the parental populations during the process of HWD. Results from this study can provide guidance on the selection of parental populations for the effective exploitation of heterosis and the prediction of genetic structure and organization for hybrid zones in nature.
\end{abstract}

Key words: Hardy-Weinberg disequilibrium, heterosis, hybrid population, outcrossing population, population genetics

\section{Introduction}

Heterosis or hybrid vigor has been one of the most important driving forces for plant breeding in the last decades (Gowen, 1952; Coors and PANDEY, 1999). The exploitation of heterosis in an operational breeding program can be made more efficient if its underlying genetic basis is known. Tremendous effort, using quantitative and molecular genetic approaches, has been expended to study the genetic causes of heterosis and its developmental and biochemical foundations (WRIGHT, 1922; Williams, 1959; SCHNELl and Cockerham, 1992; Stuber et al., 1992; XIAO et al., 1995; LI and WU, 1996; YU et al., 1997; XU and ZHU, 1999). From these studies, three major genetic hypotheses have been proposed to explain heterosis in terms of quantitative effects of genes: dominant, overdominant and epistatic (CROw, 1952, 1993, 1999; GoODNIGHT, 1999). However, the majority of these studies have been based on well designed pedigrees derived from two complementary inbred lines (homozygous) between which a simple genetic segregation pattern exists, thus facilitating a quantitative genetic analysis of heterosis (FALCONER and MACKAY, 1996).

For many outcrossing species, such as forest trees, it is very difficult or impossible to develop inbred lines due to their typical biological properties. Thus, genetic studies for these species should be based on non-domesticated natural systems. In many of these species, remarkable heterosis may be generated for a variety of morphological or life-history traits through natural or artificial hybridization between wild populations (Wu et al., 1992; Li and Wu, 1996; SteTtLeR et al., 1996; RIESEBERG, 1997).

1) College of Life Sciences, Zhejiang Forestry University, Lin'An Zhejiang 311300, P.R. China

2) Department of Statistics, University of Florida, Gainesville, FL 32611, USA

*) Author for correspondence: Rongling Wu, Department of Statistics, 533 McCarty Hall C, University of Florida, Gainesville, FL 32611, Tel \#: (352)392-3806, Fax: (352)392-8555, E-mail: rwu@stat.ufl.edu
When two different populations are crossed to generate a new progeny population, the parental populations will certainly transmit their genes and population genetic properties of these genes into their hybrids. Therefore, for the hybrids derived from natural populations, heterosis is a property of the genes concerned as well as of populations. For this reason, the traditional genetic analysis of heterosis based purely on quantitative effects of genes, will not be enough to gain a comprehensive insight into its genetic basis.

The population properties of genes have been defined with respect to allele frequency and allele association (FALCONER and MACKAY, 1996). In a large randomly mating population without selection, mutation, or migration, different alleles for the same gene are independent of each other, and the allele frequencies and genotype frequencies are constant from generation to generation. This is known as the Hardy-Weinberg law. A population composed of independent alleles for the same gene is said to be in Hardy-Weinberg equilibrium (HWE), a major assumption for most quantitative genetic studies. However, there are also other types of populations common in nature and plant breeding for which HWE may not apply. In order to study and exploit heterosis for such populations, which are said to be in Hardy-Weinberg disequilibrium (HWD), it is necessary to reveal their population genetic properties and the effects of these properties on heterosis. In this paper, we will numerically analyze how allele frequencies and HWD in the parental populations interact with gene effects to affect the direction and magnitude of heterosis as a whole.

\section{Genetic model}

Consider two different diploid populations in which there is the same segregating gene of two alleles $A_{1}$ and $A_{2}$ affecting a quantitative trait. The values relative to some zero point of the three different genotypes $A_{1} A_{1}$, and $A_{2} A_{2}$ at this locus are denoted by $\alpha, \delta$ and $-\alpha$, which assumes that $A_{1}$ and $A_{2}$ are the favorable and unfavorable alleles, respectively. This gene is supposed to have different population properties between the two populations. The allele frequencies of $A_{1}$ and $A_{2}$ in population 1 , denoted by $p_{1}$ and $q_{1}\left(p_{1}+q_{1}=1\right)$, may be different from those in population 2 , denoted by $p_{2}$ and $q_{2}\left(p_{2}+q_{2}=1\right)$. The two alleles at this locus may be genetically associated due to the influences of various evolutionary forces, with the extent described by the coefficient of Hardy-Weinberg disequilibrium (HWD). These coefficients are denoted by $d_{1}$ for population 1 and $d_{2}$ for population 2 , and have respective bounds as functions of the allele frequencies (WEIR, 1996),

$$
\left\{\begin{array}{l}
\max \left(-p_{1}^{2},-q_{1}^{2}\right) \leq d_{1} \leq p_{1} q_{1} \\
\max \left(-p_{2}^{2},-q_{2}^{2}\right) \leq d_{2} \leq p_{2} q_{2}
\end{array}\right.
$$

A negative disequilibrium coefficient corresponds to a deficiency of homozygotes relative to HWE, whereas a positive coefficient corresponds to an excess of homozygotes (HERNANDEZ and WEIR, 1989). Given these definitions, genotype frequencies for the gene in the populations are expressed by the corresponding formulae in Table 1. It is straightforward to cal- 
Table 1. - Genotypic values and frequencies of the gene under consideration in two populations.

\begin{tabular}{lccc}
\hline & & \multicolumn{2}{c}{ Genotype frequency } \\
\cline { 3 - 4 } Genotype & Genotypic value & Population 1 & Population 2 \\
\hline$A_{1} A_{1}$ & $\alpha$ & $p_{1}^{2}+d_{1}$ & $p_{2}^{2}+d_{2}$ \\
$A_{1} A_{2}$ & $\delta$ & $2\left(p_{1} q_{1}-d_{1}\right)$ & $2\left(p_{2} q_{2}-d_{2}\right)$ \\
$A_{2} A_{2}$ & $-\alpha$ & $q_{1}^{2}+d_{1}$ & $q_{2}^{2}+d_{2}$ \\
\hline
\end{tabular}

culate the means of the trait for the gene in population 1 and 2 as $\mu_{1}=\alpha\left(p_{1}-q_{1}\right)+2 \delta\left(p_{1} q_{1}-d_{1}\right)$ and $\mu_{2}=\alpha\left(p_{2}-q_{2}\right)+2 \delta\left(p_{2} q_{2}-d_{2}\right)$, respectively.

\section{Hybrid mean}

A random sample of individuals from one population as the female parents, is crossed with a random sample of individuals from the other population, the male parents, to produce an inter-population hybrid progeny population. Because the two samples used as parents represent a random sample of each original population, the population genetic parameters in the original populations, such as allele frequency and HWD coefficient, can be approximated by those in the samples. Thus, genotype frequencies in the hybrid population can be estimated based on the parameters defined for the original populations. Assuming that each genotype for the locus has an equal probability of survival in the hybrid population, i.e., the hybridization does not cause new HWD, the frequencies of the three genotypes in the hybrid population are:

$$
\begin{aligned}
P_{11}= & \left(p_{1}^{2}+d_{1}\right) \times\left(p_{2}^{2}+d_{2}\right)+\frac{1}{2}\left(p_{1}^{2}+d_{1}\right) \times 2\left(p_{2} q_{2}-d_{2}\right) \\
& +\frac{1}{2} \times 2\left(p_{1} q_{1}-d_{1}\right) \times\left(p_{2}^{2}+d_{2}\right)+\frac{1}{4} \times 2\left(p_{1} q_{1}-d_{1}\right) \times 2\left(p_{2} q_{2}-d_{2}\right) \\
= & p_{1} p_{2}
\end{aligned}
$$

for genotype $A_{1} A_{1}$,

$$
\begin{aligned}
P_{12}= & \frac{1}{2}\left(p_{1}^{2}+d_{1}\right) \times 2\left(p_{2} q_{2}-d_{2}\right)+\left(p_{1}^{2}+d_{1}\right)\left(q_{2}^{2}+d_{2}\right)+\frac{1}{2} \times 2\left(p_{1} q_{1}-d_{1}\right) \times\left(p_{2}^{2}+d_{2}\right) \\
& +\frac{1}{2} \times 2\left(p_{1} q_{1}-d_{1}\right) \times 2\left(p_{2} q_{2}-d_{2}\right) \\
& +\frac{1}{2} \times 2\left(p_{1} q_{1}-d_{1}\right)\left(q_{2}^{2}+d_{2}\right)+\left(q_{1}^{2}+d_{1}\right)\left(p_{2}^{2}+d_{2}\right)+\frac{1}{2}\left(q_{1}^{2}+d_{1}\right) \times 2\left(p_{2} q_{2}-d_{2}\right) \\
= & p_{1} q_{2}+q_{1} p_{2}
\end{aligned}
$$

for genotype $A_{1} A_{2}$, and

$$
\begin{aligned}
P_{22}= & \left(q_{1}^{2}+d_{1}\right) \times\left(q_{2}^{2}+d_{2}\right)+\frac{1}{2}\left(q_{1}^{2}+d_{1}\right) \times 2\left(p_{2} q_{2}-d_{2}\right) \\
& +\frac{1}{2} \times 2\left(p_{1} q_{1}-d_{1}\right) \times\left(q_{2}^{2}+d_{2}\right)+\frac{1}{4} \times 2\left(p_{1} q_{1}-d_{1}\right) \times 2\left(p_{2} q_{2}-d_{2}\right) \\
= & q_{1} q_{2}
\end{aligned}
$$

for genotype $A_{2} A_{2}$.

It can be seen that genotype frequencies in the hybrid progeny population are independent of the HWD coefficient in both original populations. In nature, when any two different populations are merged, the new population will reach HWE in one generation. For this reason, hybrid zones occurring between two different but adjacent species must be in HWE as long as they are not subject to new evolutionary forces. This is the extension of the HWE law to different populations.

The trait mean of the hybrid population for the gene can be expressed as $M=\alpha\left(p_{1} p_{2}-q_{1} q_{2}\right)+\delta\left(p_{1} q_{2}-q_{1} p_{2}\right)$. According to the traditional definition, heterosis is calculated by comparing hybrids with inbred parents. But here heterosis is defined in terms of the performance of outcrossing parental populations (FALCONER and MACKAY, 1996), calculated as the merit of the hybrid population over the average value of the two parental populations $\left(H_{A}\right)$ or the better parental populations $\left(H_{B}\right)$ :

$$
\begin{aligned}
H_{A} & =M-\frac{1}{2}\left(\mu_{1}+\mu_{2}\right) \\
& =\alpha\left\{\left(p_{1} p_{2}-q_{1} q_{2}\right)-\frac{1}{2}\left[\left(p_{1}-q_{1}\right)+\left(p_{2}-q_{2}\right)\right]\right\}+\delta\left[\left(q_{2}-q_{1}\right)\left(p_{1}+p_{2}\right)+d_{1}+d_{2}\right]
\end{aligned}
$$

$$
\begin{aligned}
H_{B} & =M-\max \left\{\mu_{1}, \mu_{2}\right\} \\
& =\alpha\left(q_{1} p_{2}-p_{1} q_{2}\right)+\delta\left[\left(q_{2}-q_{1}\right)\left(p_{1}-q_{1}\right)+2 d_{1}\right]
\end{aligned}
$$

It can be seen that the additive $(\alpha)$ and dominant genetic effects $(\delta)$ affect heterosis through different mechanisms. Whereas the influence of $\alpha$ depends on the degree of the fixture of alleles in the parental populations, the influence of $\delta$ depends on the difference of allele frequencies between the populations and the genetic association (measured by $d_{1}$ and $d_{2}$ ) between the two alleles for the gene. In Eq. (3), we assume that population 1 is better than population 2 , which implies $\mu_{1}-\mu_{2}=2\left[\alpha\left(p_{1}-p_{2}\right)+\delta\left(p_{1} q_{1}-d_{1}-p_{2} q_{2}+d_{2}\right)\right]>0$, resulting in particular restrictions on the relationship between the allele frequencies of the two populations (see the Results).

\section{Hybrid genetic variance}

The genetic variance due to the gene in a parental population (say i) can be derived as

$$
\begin{aligned}
\sigma_{g_{i}}^{2} & =2\left[\left(p_{i} q_{i}+d_{i}\right) \alpha^{2}+\left(p_{i} q_{i}-d_{i}\right)\left(1-2 p_{i} q_{i}+2 d_{i}\right) \delta^{2}-2\left(p_{i}-q_{i}\right)\left(p_{i} q_{i}-d_{i}\right) \alpha \delta\right] \\
& \left.=\sigma_{a_{i}}^{2}+\sigma_{d_{i}}^{2}+2 d_{i} \lambda_{i}^{2}+2\left(q_{i}-p_{i}\right)\left(p_{i} q_{i}-2 d_{i}\right)\left(\lambda_{i}+\alpha\right) \delta\right]-4\left(\delta d_{i}\right)^{2}
\end{aligned}
$$

where $\sigma_{a_{i}}^{2}=2 p_{i} q_{i} \alpha_{i}^{2}, \sigma_{d_{i}}^{2}=4\left(p_{i} q_{i} \delta\right)^{2}$ and $\lambda_{\mathrm{i}}=\alpha+\left(q_{i}-p_{i}\right) \delta$ are the additive genetic variance, dominant genetic variance and the average effect of the gene substitution at the locus concerned, respectively, when the population is in HWE (FALCONER and MACKAY, 1996). It is seen from Eq. (4) that the genetic variance of a population in HWD is not simply the sum of the additive and dominant genetic variances. It additionally contains interactions between gene effects, allele frequency and coefficients of HWD. These additional interactions will not exist if the population is in HWE $\left(d_{i}=0\right)$.

The difference in genetic variance between two populations is dependent on how allele frequencies and HWDs differ and how these population parameters and gene effects interact, as shown by the following expression:

$$
\begin{aligned}
\psi_{g}^{2}= & \sigma_{g_{1}}^{2}-\sigma_{g_{2}}^{2} \\
= & 2\left[\left(p_{1}-p_{2}\right)\left(1-p_{1}-p_{2}\right)+\left(d_{1}-d_{2}\right)\right] \alpha^{2} \\
& +2\left\{\left(p_{1}-p_{2}\right)\left(1-p_{1}-p_{2}\right)\left[1-2\left(p_{1}+p_{2}\right)+2\left(p_{1}^{2}+p_{2}^{2}\right)\right]\right. \\
& \left.+4\left[\left(p_{1} d_{1}-p_{2} d_{2}\right)-\left(p_{1}^{2} d_{1}-p_{2}^{2} d_{2}\right)\right]-\left(d_{1}-d_{2}\right)\left(1-2 d_{1}-2 d_{2}\right)\right\} \delta^{2} \\
& -4\left[\left(p_{1}-p_{2}\right)\left(1+2 p_{1}^{2}+2 p_{1} p_{2}+2 p_{2}^{2}-3 p_{1}-3 p_{2}\right)+2\left(p_{1} d_{1}-p_{2} d_{2}\right)-\left(d_{1}-d_{2}\right)\right] \alpha \delta
\end{aligned}
$$

When the two populations with different genetic structures are crossed, the genetic variance due to the gene in the hybrid population $\left(\delta_{G}^{2}\right)$ can be derived as

$$
\begin{aligned}
\sigma_{G}^{2}= & {\left[p_{1} p_{2}\left(1-p_{1} p_{2}+q_{1} q_{2}\right)+q_{1} q_{2}\left(1+p_{1} p_{2}-q_{1} q_{2}\right)\right] \alpha^{2} } \\
& +\left(p_{1} q_{2}+q_{1} p_{2}\right)\left(1-p_{1} q_{2}-q_{1} p_{2}\right) \delta^{2} \\
& -2\left(p_{1} p_{2}-q_{1} q_{2}\right)\left(p_{1} q_{2}+q_{1} p_{2}\right) \alpha \delta,
\end{aligned}
$$

which indicates that the traditional partitioning of genetic variance into additive and dominant components is not possible for the hybrid populations.

\section{Results}

In this section, we will use numerical examples to demonstrate how allele frequencies and HWD affect heterosis and genetic variation in the hybrid progeny. For the effect of HWD, we consider three different cases, coefficient of HWD $=0$ and HWD on the two extremes as shown by Eq. (1).

\section{Mid-parent heterosis}

As described by Eq. (2), heterosis based on the mid-parent $\left(H_{A}\right)$ is independent of the additive effect of the gene. Assume that the dominant effect $\delta=10$. It is obvious that the magnitude of $H_{A}$ is a function of the difference in allele frequency 
A

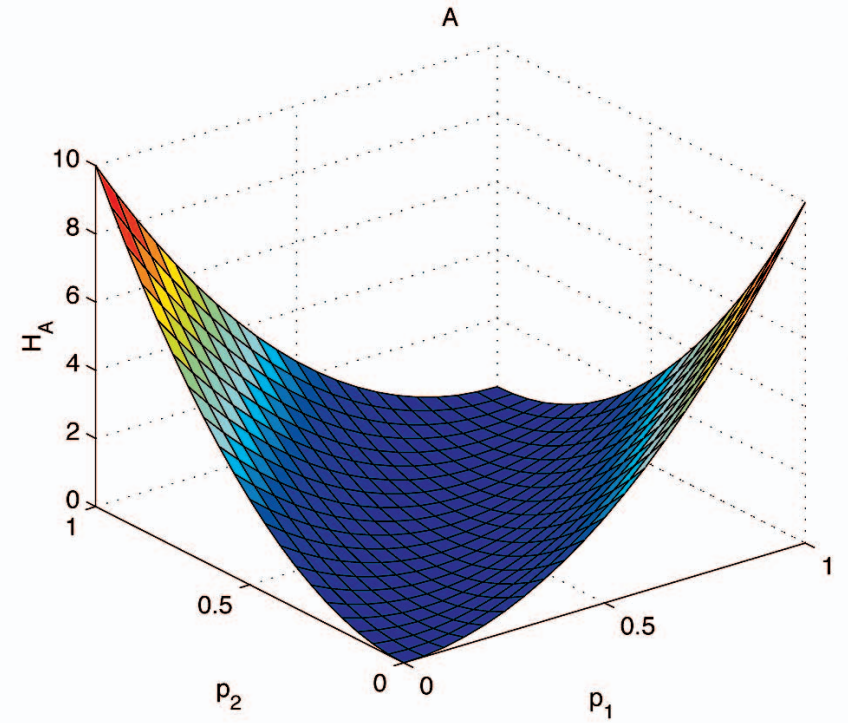

C

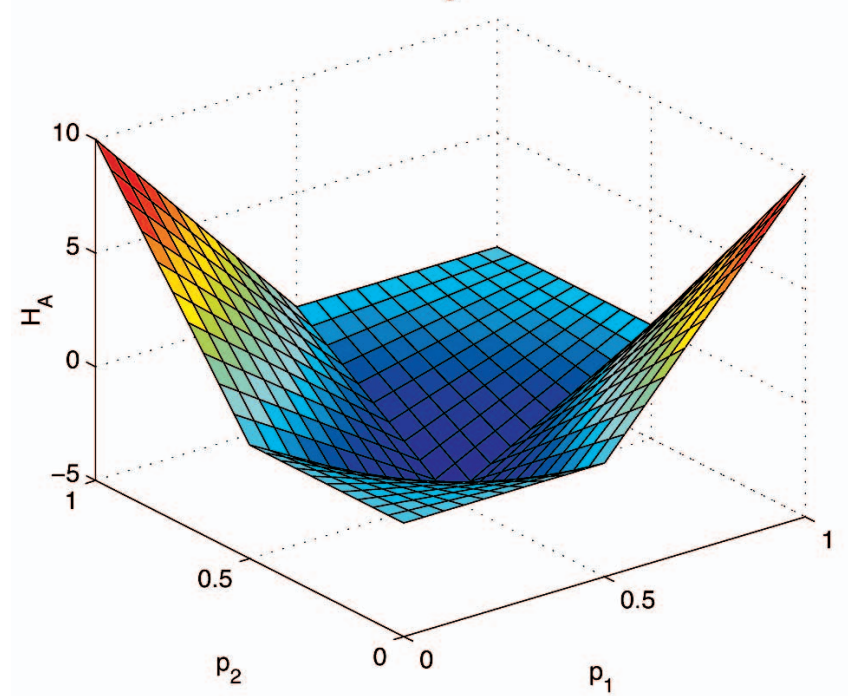

B

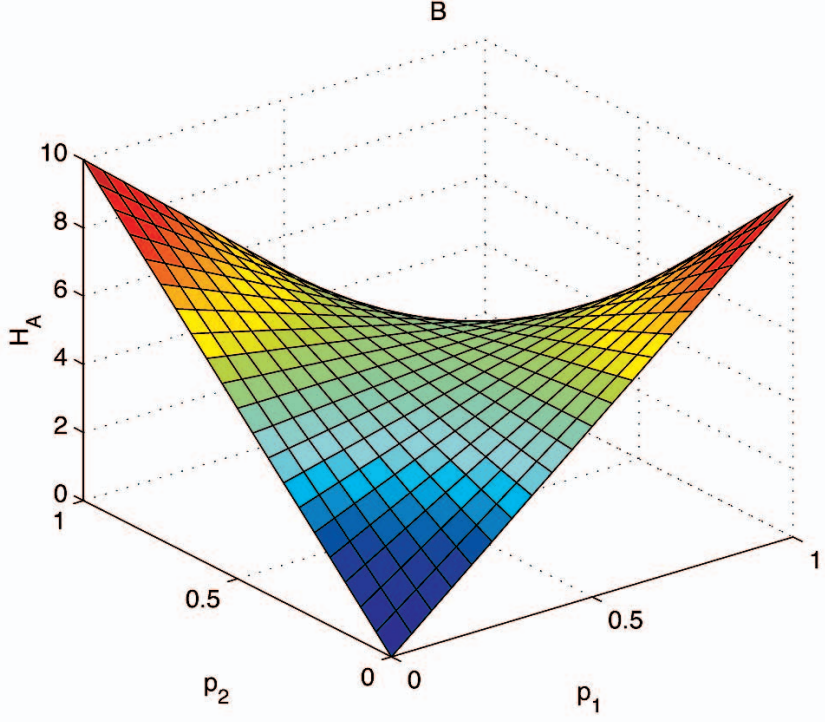

Figure 1. - Plots of mid-parent heterosis vs. allele frequencies in two parental populations. A: Populations in HWE, B: Populations with HWD at upper bound, $C$ : Populations with HWD at the lower bound.

between the two parental populations (Figs. 1A-1C). When the favorable allele is fixed in one population and the unfavorable allele is fixed in the second (i.e., $p_{1}=1$ and $p_{2}=0$ or $p_{1}=0$ and $p_{2}=1$ ), the mid-parent heterosis is maximized. There is no heterosis when a given allele has the same frequency in the two populations.

The magnitude of $H_{A}$ also differs depending on whether or not there is HWD (Figs. $1 \mathrm{~A}$ vs. $1 B$ and $1 C$ ) and how much HWD is in the two parental populations (Fig. $1 B$ vs. $1 C$ ). Given the same difference between allele frequencies (except those at the extremes) of the two populations, larger positive $H_{A}$ is observed in the case where HWD is at the upper bound (Fig. $1 B$ ) than in the case where there is no HDW (Fig. 1A) or where HWD is at the lower bound (Fig. 1C). In the case in which HDW is at the lower bound, $H_{A}$ may be negative when the allele frequencies tend to be identical between the two populations.

\section{Better-parent heterosis}

The influences of allele frequencies and HWD on heterosis based on the better parent $\left(H_{B}\right)$ are compared. This is because the direction and size of $H_{B}$ is dependent of the degree of dominance as described by the ratio of $\delta$ to $\alpha$ and because there are restrictions on the relationship between the allele frequencies of the two populations. Assume that population 1 is the better parent. Thus, when there is no HWD in both parental populations, the value of $p_{2}$ should be $0 \leq p_{2} \leq p_{1} \leq 1$ for a dominant or partially dominant gene and

$$
\begin{cases}0 \leq p_{1} \leq p_{2} \leq 1 \text { or } 0 \leq p_{2} \leq \frac{\alpha}{\delta}+q_{1} & \text { if } p_{1}-q_{1}>\frac{\alpha}{\delta} \\ 0 \leq p_{2} \leq p_{1} \leq 1 \text { or } 0 \leq \frac{\alpha}{\delta}+q_{1}<p_{2} \leq 1 & \text { if } p_{1}-q_{1}<\frac{\alpha}{\delta} \\ 0 \leq p_{1} \leq p_{2} \leq 1 \text { or } 0 \leq p_{2}<p_{1} \leq 1 & \text { if } p_{1}-q_{1}=\frac{\alpha}{\delta}\end{cases}
$$

for an overdominant gene. When HWDs in both populations are at the upper bound, the value of $p_{2}$ is $0 \leq p_{2} \leq p_{1} \leq 1$, irrespective of the dominance of the gene. When HWDs in both populations are at the lower bound, the value of $p_{2}$ will be taken as:

$\begin{cases}0 \leq p_{2}<p_{1} \text { or } 0.5 \leq p_{2}<\frac{\alpha p_{1}-\delta q_{1}}{\alpha-\delta}(\alpha>\delta) \text { or } \frac{\alpha p_{1}-\delta q_{1}}{\alpha-\delta}<p_{2}(\alpha<\delta) & \text { if } 0 \leq p_{1} \leq 0.5 \\ 0.5 \leq p_{2}<p_{1} \text { or } 0 \leq p_{2}<1-\frac{\delta p_{1}}{\alpha+\delta} & \text { if } 0.5<p_{1} \leq 1\end{cases}$

As demonstrated by the numerical analyses, $H_{B}$ increases with the difference of allele frequencies between the two 
parental populations, with the extent of increase depending on the degrees of HWD and dominance (results not shown). Under the same degree of dominance, larger $H_{B}$ can be generated for two parental populations with HWDs at the upper bound than the two populations without HWDs. At a given HWD level, dominance increases the heterosis significantly. However, the heterosis is affected by interactions between HWD and dominance. The effect of HWD on the heterosis is increased from partial dominance $(\delta / \alpha=0.5)$ to dominance $(\delta / \alpha=1.0)$ to overdominance $(\delta / \alpha=1.5)$. For example, at partial dominance, the difference of the heterosis between the situation in which there is HWD at the upper bound and the situation of no HWD is 0.9. This difference is increased to 2.4 at dominance $(\delta / \alpha=1)$ and 2.7 at overdominance $(\delta / \alpha=1.5)$.

When the two parental populations have HWDs at the lower bound, the effect of the difference of allele frequency on $H_{B}$ has different trends for overdominance $(\delta / \alpha=1.5)$. In this example, the heterosis has a minimum value at $p_{1}=0.5$. When $p_{1}$ decreases from 0.5 to 0 or increases from 0.5 to 1 , the heterosis is increased. In all cases, the heterosis is increased with increased $p_{2}$ values, although it is negative for many combinations of $p_{1}$ and $p_{2}$.
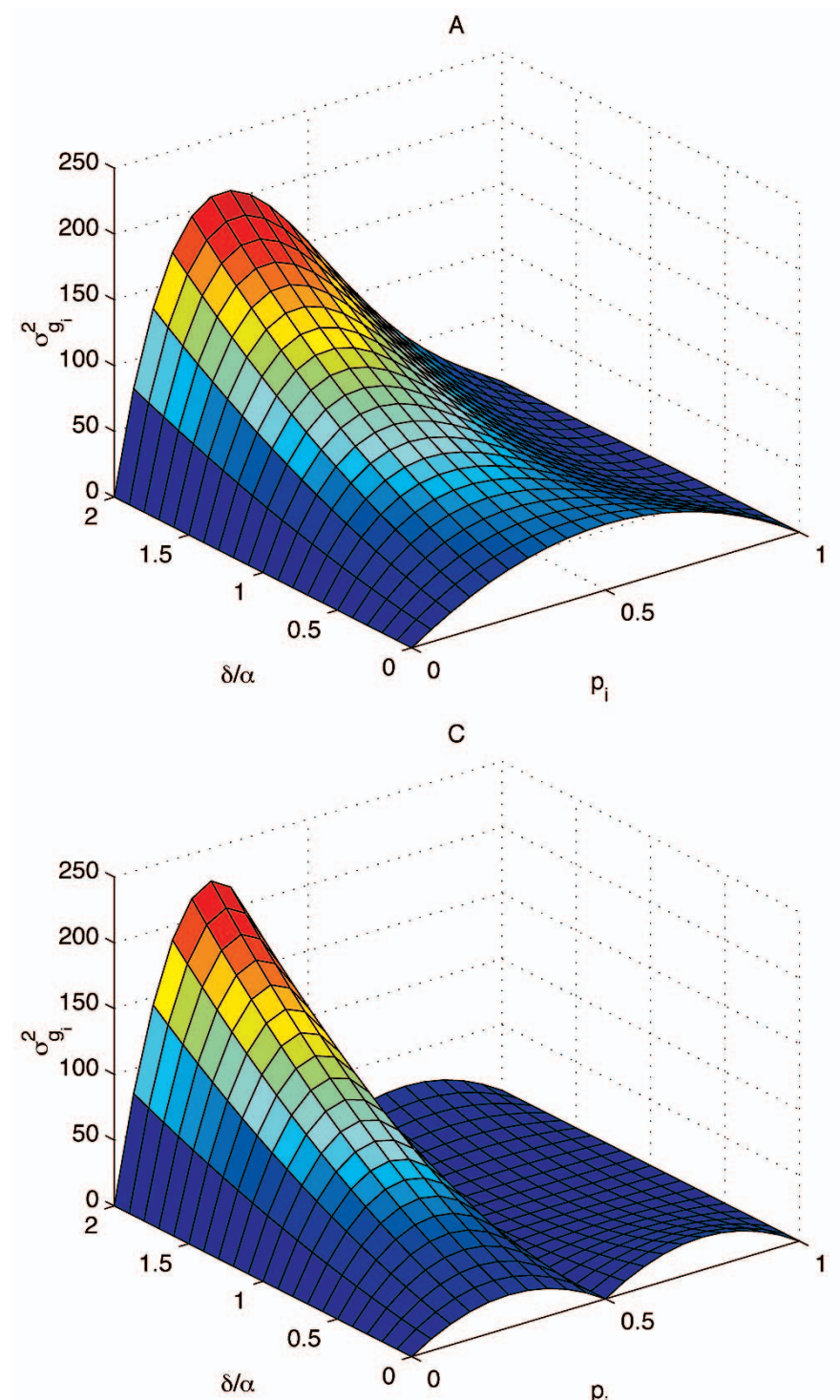

\section{Population difference and hybrid population}

The genetic variance of a parental population is a function of the difference of the frequencies of alternative alleles, the degree of HWD and the degree of dominance (Fig. 2). In a HWE population, the genetic variance is increased consistently with increased divergence in the frequencies of alternative alleles and increased dominance (Fig. 1A). However, for a population with HWD at the upper bound, the increase of the genetic variance with the difference of the alternative allele frequencies is not dependent on the degree of dominance (Fig. 2B), because there are no heterozygotes in this case. For a population with HWD at the lower bound, there exist two distinct trends for the genetic variance (Fig. 2C). If the frequency of the favorable allele ranges from 0 to 0.5 , the pattern of the values for the genetic variance is the same as for a population without HWD. But if the allele frequency ranges from 0.5 to 1 , the pattern of the values for the genetic variance is the same as for a population with HWD at the upper bound.

As expected, the difference in genetic variance between two natural populations depends on how the allele frequencies and HWD differ between the populations (see Eq. 5). These two population parameters further interact with the degree of dom-

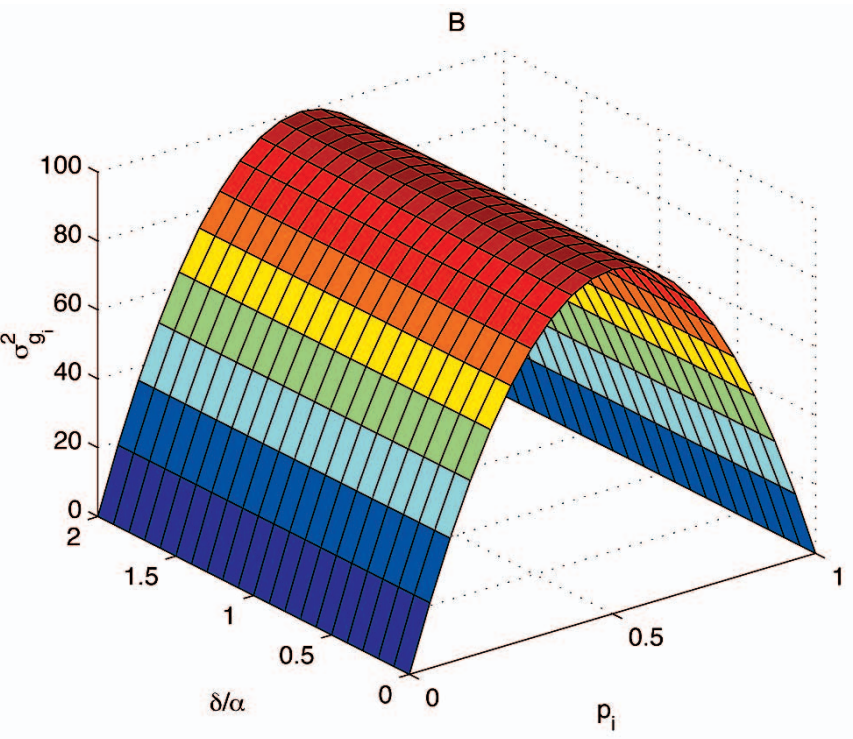

Figure 2. - Plots of genetic variance vs. allelic frequency and the degree of dominance for a natural population. A: Population in HWE, B: Population with HWD at the upper bound, $C$ : Population with HWD at the lower bound. 
A
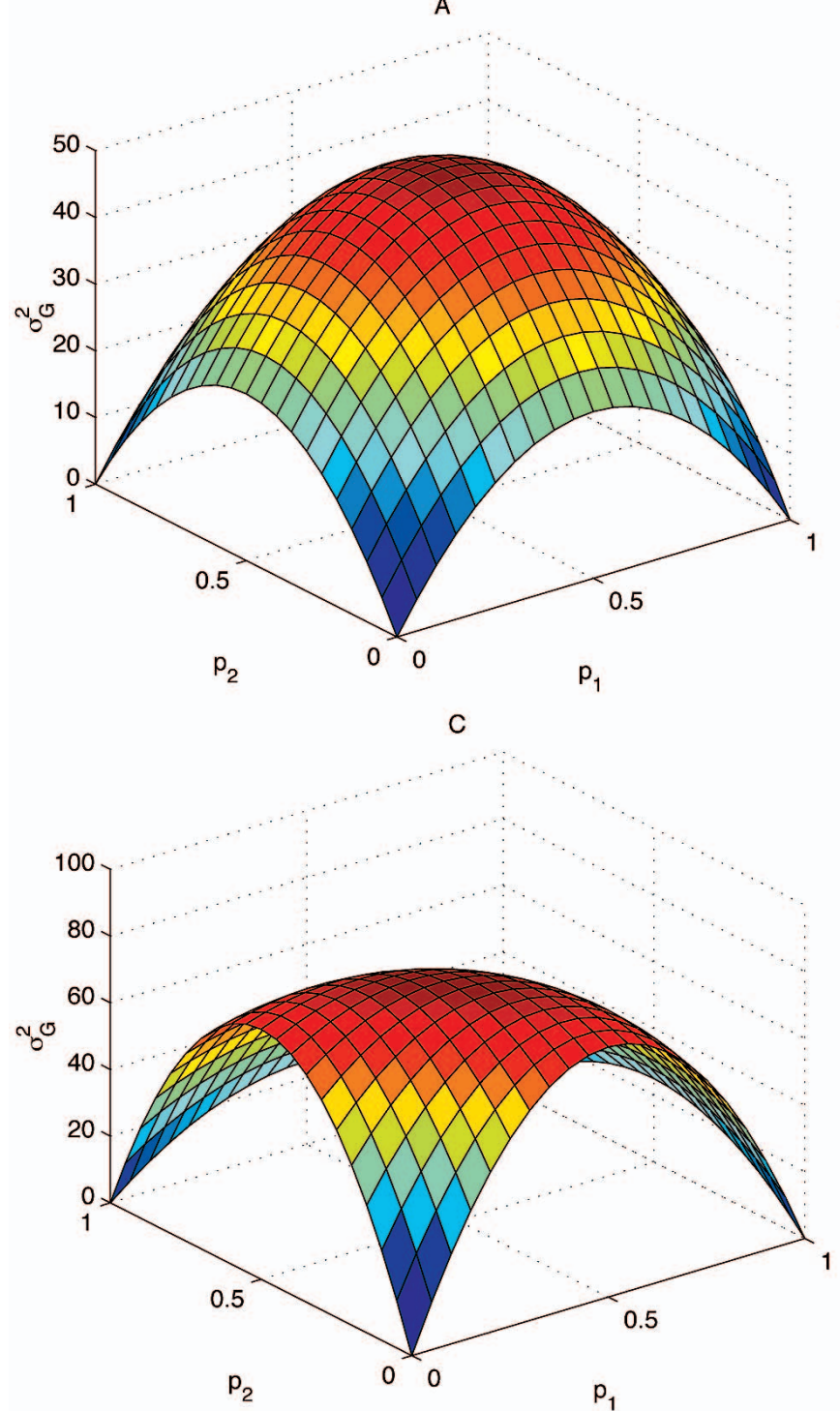

B

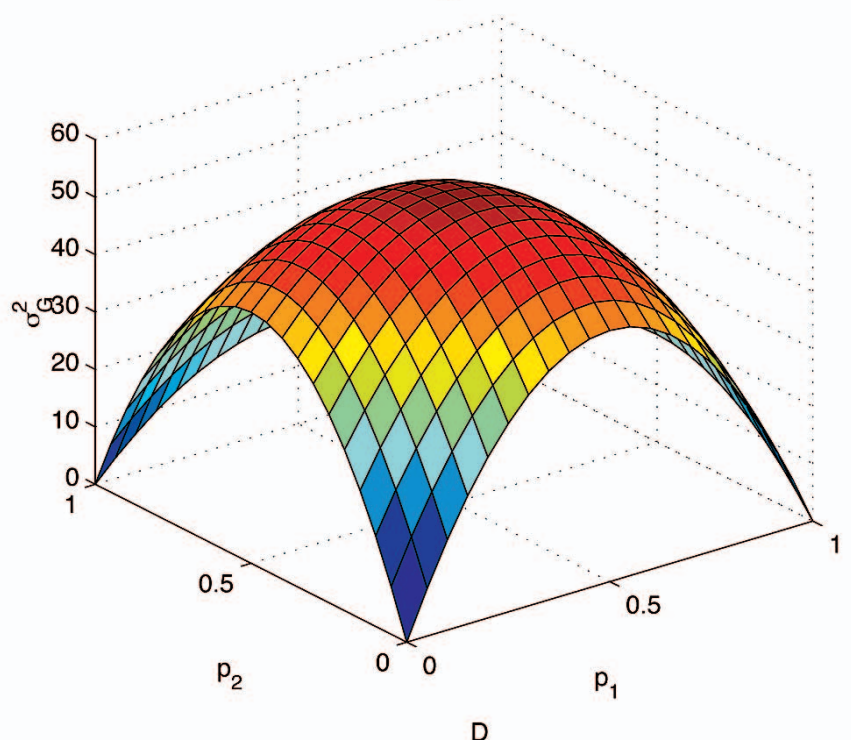

D

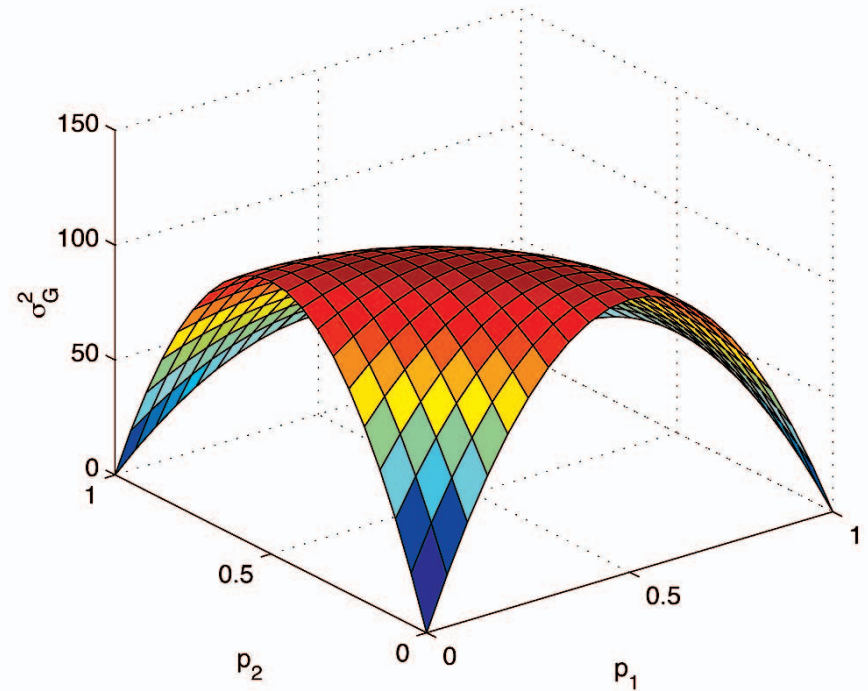

Figure 3. - Plots of genetic variance in the hybrid population vs. allelic frequencies in two parental populations under pure additivity (A), partial dominance $(B)$, dominance $(C)$ and overdominance $(D)$.

inance to affect the population difference of genetic variance. The genetic variance of the hybrid population is not dependent on HWD, but is affected by the difference of the frequency of the same allele between the two populations. If the allele frequency is $1 / 2$ in both populations, the genetic variance of the hybrid population is maximum (Fig. 3). The genetic variance of the hybrid population is a function of the degree of dominance, increasing from no dominance $(\delta / \alpha=0, F i g$. $3 A$ to partial dominance $(\delta / \alpha=0.5, F i g .3 B$ to dominance $(\delta / \alpha=1$, Fig. $3 C)$ to overdominance $(\delta / \alpha=1.5$, Fig. $3 D)$.

\section{Discussion}

Traditional genetic analyses of heterosis are based on the quantitative effects of the genes segregating between two complementary opposite inbred lines. Three genetic hypotheses, dominance, overdominance, or epistasis, have been extracted from these analyses to explain the genetic mechanisms of heterosis. However, heterosis for the hybrids derived from two different outcrossing populations, especially those in HWD, is not only dependent on gene effects (this is the essence of quantitative genetics) but also the genetic structures and evolutionary histories of the populations (this is the essence of population genetics). One of the main motivations for this study is to investigate the genetic basis of heterosis from both quantitative and population genetic perspectives. It is found that heterosis based on either the mid-parent or better parent is affected by genetic divergence in allele frequency and allele association between two populations. These population parameters interact with gene effects and gene actions to determine heterosis.

There have been a number of well documented examples showing that heterosis is increased by the genetic diversity between two populations (COX and MURPHY, 1990; LABATE et al., 1998). Increased heterosis with population divergence has often been attributed to the difference of allele frequencies between populations (CRESS, 1966). Our theoretical analyses indicate that the disequilibrium status of populations can also offer a mechanistic explanation for heterosis in natural populations. Although fundamental theories of quantitative genetics are based on the properties of a large, randomly mating population (FALCONER and MACKAY, 1996), deviations from HWE have not been uncommon in many situations for domesticated and wild species. The generation of HWD may be due to a number of evolutionary forces, such as selection, mutation, migration and subdivision (FALCONER and MACKAY, 1996). 
Two populations that are in HWD can reach HWE when they are randomly mated for one generation. This may suggest that there is a higher probability for hybrid zones to be in HWD than either parental population in nature, before new evolutionary forces have an effect on the hybrid population. However, occurrence of HWD in the parental population affects the magnitude of heterosis in the hybrid population. Consider two pairs of outcrossing populations, one pair in HWD and the other in HWE. Any population from one pair has the same allele distributions as a population from the other pair. As illustrated in this study, the mating of the pair of populations in HWD will produce extreme heterosis compared to the mating of the other pair of populations in HWE. According to LAMKEY and EDWARDS (1999), we define the heterosis of the randomly mating populations in HWE as panmictic heterosis. The "extreme" heterosis between the two populations in HWD is a kind of baseline heterosis, as defined by LAMKEY and EDWARDS (1999), which is the restoration of what was lost in the parental populations because of HWD. Our analysis is based on the assumption that new HWD is created during hybridization between two outcrossing populations. But the generation of HWD for the hybrids is not imposssible because of possible outcrossing depression. It is straightforward to incorporate the effect of HWD resulting from hybridization in our heterosis analysis.

Hybrid populations with strong heterosis may be an important force for speciation (RIESEBERG, 1997). New developments of quantitative genetic studies of hybrid populations like those in this study are appealing, because many quantitative genetic concepts such as average effect, additive genetic variance and narrow sense heritability are inappropriate for understanding the genetic organization and structure of the hybrid population (GORDON, 1999).

\section{Acknowledgements}

We thank anonymous referees for their constructive comments on this manuscript. This work is partially supported by an Outstanding Young Investigators Award (No. 30128017) of the National Natural Science Foundation of China and the University of Florida Research Opportunity Fund (No. 02050259) to R. W. This manuscript was approved for publication as Journal Series No. R-10061 by the Florida Agricultural Experiment Station.

\section{References}

Coors, J. G. and PAndey, S.: The genetics and exploitation of heterosis in crop plants. Crop Science Society of America and American Society of Agronomy, Madison, WI (1999). - Cox, T. S. and Murphy, J. P.: The effect of parental divergence on heterosis in winter wheat crosses. Theor Appl Genet 79: 241-250 (1990). — CRESS, C. E.: Heterosis of the hybrid related to gene frequency differences between two populations. Genetics 53: 269-274 (1966). - CROw, J. F.: Dominance and overdominance. In J. W. Gowen (ed.). Heterosis, pp. 282-297. Iowa State College Press, Ames, IA (1952). - CROw, J. F.: Mutation, mean fitness, and genetic load. Evol Biol 9: 3-42 (1993), - CRow, J. F. Dominance and overdominance. In: Coors J. G., PANDEY, S. (eds.). The genetics and exploitation of heterosis in crop plants, pp. 49-58. Crop Science Society of America and American Society of Agronomy, Madison, WI (1999). — FALCONER D. S. and MackAY, T. F. C.: Introduction to quantitative genetics. 4th Edn. Longman Sci. and Tech., Harlow, UK (1996). - Goodnight, C. J.: Epistasis and heterosis. In: Coors, J. G., PANDEY, S. (eds.). The genetics and exploitation of heterosis in crop plants, pp. 59-68. Crop Science Society of America and American Society of Agronomy, Madison, WI (1999). - GoRDON, I. L.: Quantitative genetics of intraspecific hybrids. Heredity 83: 757-764 (1999). - GowEN, I. W.: Heterosis. Iowa State College Press, Ames, IA (1952). - Hernandez, J. L. and Weir, B. S.: A disequilibrium coefficient approach to Hardy-Weinberg testing. Biometrics 45: 53-70 (1989). - Lebate, J. A., LAmkey, K. R., Lee, M. and Woodman, W. L.: Population genetics of increased hybrid performance between two maize populations under reciprocal recurrent selection. In: Coors, J. G., PANDEY, S. (eds.). The genetics and exploitation of heterosis in crop plants, pp. 127-137. Crop Science Society of America and American Society of Agronomy, Madison, WI (1999). - LAMkEY, K. R. and EDWARDS, J. W.: Quantitative genetics of heterosis. In: CooRs, J. G., PANDEY, S. (eds.). The genetics and exploitation of heterosis in crop plants, pp. 31-48. Crop Science of America and American Society of Agronomy, Madison, WI (1999). - LI, B. and WU, R.: Genetic causes of heterosis in juvenile aspen: a quantitative comparison across intra- and interspecific crosses. Theor Appl Genet 93: 380-391 (1996). — LI, C. C. Pseudo-random mating population. In celebration of the 80th anniversary of the Hardy-Weinberg law. Genetics 119: 731-737 (1988). — RIESEBERG, L. H.: Hybrid origins of plant species. Annu Rev Ecol Syst 28: 359-389 (1997). - Schnell, F. W. and CockerhaM, C. C.: Multiplicative vs. arbitrary gene action in heterosis. Genetics 131: 461-469 (1992). STETTLER, R. F., Zsuffa, L. and Wu, R.: The role of hybridization in the genetic manipulation of Populus. In: SteTtler, R. F., Bradshaw, H. D. Jr., Heilman, P. E., Hinckley, T. M. (eds.). Biology of Populus and its implications for management and conservation, pp 87-112. NRC Research Press, National Research Council of Canada, Ottawa, ON (1996). - Stuber, C. W., Lincoln, S. E., Wolff, D. W., Helentuaris, T. and LANDER, E. S.: Identification of genetic factors contributing to heterosis in a hybrid from two elite maize inbred lines using molecular markers. Genetics 132: 823-839 (1992). - WEIR, B. S.: Genetic data analysis II Sinauer Associates, Sunderland, MA (1996). - Williams, W.: Heterosis and the genetics of complex characters. Nature 184: 527-530 (1959). WRIGHT, S.: The effect of inbreeding and crossbreeding on guinea pigs. III. Crosses between highly inbred families. Tech Bull USDA 1121, 60 pp (1922). - Wu, R. L., WANG, M. X. and HuANG, M. R.: Quantitative genetics of yield breeding for Populus short rotation culture. I. Dynamics of genetic control and selection models of yield traits. Can J For Res 22: 175-182 (1992). — XIAO, J., LI, J., YuAn, L. and TANKSLEY, S. D. Dominance is the major genetic basis of heterosis in rice as revealed by QTL analysis using molecular markers. Genetics 140: 745-754 (1995). - XU, Z. C. and ZHU, J.: An approach for predicting heterosis based on an additive, dominance and additive $\times$ additive model with environment interaction. Heredity 82: 510-517 (1999). - YU, S. B., LI, J. X., TAN, X. F., GaO, Y. J., LI, X. H., ZhANG, Q. and Maroof, M. A. S.: Importance of epistasis as the genetic basis of heterosis in an elite rice hybrid. Proc Natl Acad Sci USA 94: 9226-9231 (1997). 